\title{
Deep photometry of galaxies in the VEGAS survey: the case of NGC 4472
}

\author{
Marilena Spavone on behalf of the VEGAS team ${ }^{\star}$
}

\begin{abstract}
The VST-VEGAS project is aimed at observing and studying a rich sample of nearby early-type galaxies in order to systematically characterize their properties over a wide baseline of sizes and out to the faint outskirts where data are rather scarce so far. The external regions of galaxies more easily retain signatures about the formation and evolution mechanisms which shaped them, as their relaxation time are longer, and they are more weakly influenced by processes such as mergers, secular evolution, central black hole activity, and supernova feedback on the ISM, which tend to level age and metallicity gradients. The collection of a wide photometric dataset of a large number of galaxies in various environmental conditions, may help to shed light on these questions. To this end VEGAS exploits the potential of the VLT Survey Telescope (VST) which provides high quality images of one square degree field of view in order to satisfy both the requirement of high
\end{abstract}

* VEGAS team: M. Capaccioli (P.I.), Michele Cantiello, D. A. Forbes, A. Grado, E. Iodice, L. Limatola, N. Napolitano, M. Paolillo, T. H. Puzia, G. Raimondo, A. J. Romanowsky, P. Schipani \& M. Spavone resolution data and the need of studying nearby, and thus large, objects. We present a detailed study of the surface photometry of the elliptical galaxy NGC4472 and of smaller ETGs in its field, performed by using new $\mathrm{g}$ and $\mathrm{i}$ bands images to constrain the formation history of this nearby giant galaxy, and to investigate the presence of very faint substructures in its surroundings.

\section{The VEGAS survey}

The VST Elliptical GAlaxies Survey (VEGAS) is a deep multi-band $(g, r, i)$ imaging survey of earlytype galaxies in the Southern hemisphere carried out with VST at the ESO Cerro Paranal Observatory (Chile). The survey goal is to map the surface brightness of galaxies with $V_{\text {rad }}<4000$ $\mathrm{km} / \mathrm{s}$, sampling all environmental conditions and the whole parameter space. The expected depths at $\mathrm{S} / \mathrm{N}>3$ in the $g, r$ and $i$ bands are 27.3, 26.8, and 26 mag $\operatorname{arcsec}^{-2}$ respectively, enough to detect signatures of diffuse star components around 
galaxies (see e.g. [9]) and the dynamical interactions of ETGs with the intergalactic medium. The main aspects that the VEGAS survey will investigate are: 1) 2D light distribution out to 8-10 $R_{e}$ : galaxy structural parameters and diffuse light component, inner substructures as a signature of recent cannibalism events, inner disks and bars fueling active nuclei present in almost all the objects of our sample; 2) radially averaged surface brightness profiles and isophote shapes up to 10 $R_{e}$; 3) color gradients and the connection with galaxy formation theories; 4) detection of external low-surface brightness structures of the galaxies and the connection with the environment; 5) census of small stellar systems (SSS: GCs, ultra compact dwarfs and galaxy satellites) out to $\sim 200$ $\mathrm{kpc}$ from the main galaxy center, and their photometric properties (e.g. GC luminosity function and colors and their radial changes out to several $R_{e}$ allowing to study the properties of GCs in the outermost "fossils" regions of the host galaxy. VEGAS will provide a volume limited survey in the South, complementary to the Next generation Virgo Cluster Survey (NGVS), with comparable depth but no environmental restrictions.

\section{The NGC 4472 field: a test case}

This first VEGAS case (Capaccioli et al., in preparation) deals with a deep photometric analysis of the ETGs in the VST field of the galaxy NGC 4472 (M49), the brightest member of the Virgo cluster. This field has been chosen for the following reasons:

- it is well-studied, with an ample scientific photometric literature ([3], [8], [7], [5], [6]);

- it offers a wide range of cases where to investigate the ability of VEGAS to map the faint galaxy outskirts. In fact, together with a nearby supergiant object filling almost the entire OmegaCAM field, there are smaller ETGs either embedded in the light of NGC 4472 or close to the edges of the frame.

\subsection{Light and color distribution}

We used the standard IRAF task ELLIPSE to perform the isophotal analysis of the VEGAS galaxies on the final mosaic in each band, after proper background subtraction. We have also derived the azimuthally averaged surface brightness profiles in isophotal annuli of specified thickness. The azimuthally averaged profiles for NGC 4472 in the $g$ and $i$ bands are shown in Fig 1 as a function of the isophote semi major axis $a$.

The light profile in the $g$ band, shows a neat change in the slope at $a_{e}^{1 / 4} \simeq 5.5$, where $\mu_{g} \sim 27$ mag $\operatorname{arcsec}^{-2}$. The level at which the break occurs is compatible with the typical values at which [9] have observed a change of slope induced by the ICL in a series of stacked galaxy clusters. Moreover we note the presence of an outer and more elliptical component with a significant gradient in the P.A. which, as suggested by [4], is likely due to a population of some ICL.

Our azimuthally averaged $g$ band profile is compared with the available literature in Fig. 2. The NGC 4472

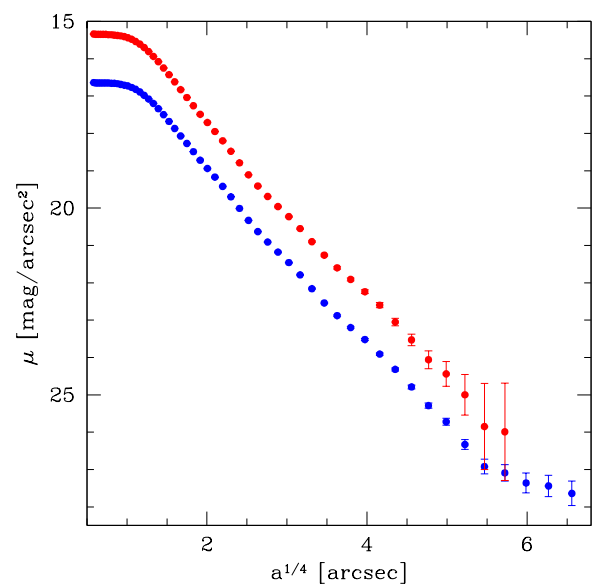

Fig. 1 Azimuthally averaged light profiles for NGC 4472 in the $g$ (blue) and $i$ (red) bands.

${ }^{2}$ IRAF is distributed by the National Optical Astronomy Observatories, which is operated by the Associated Universities for Research in Astronomy, Inc. under cooperative agreement with the National Science Foundation.(Image Reduction and Analysis Facility) environment. 
residuals with respect to $r^{1 / 4}$ fits show a spectacular agreement with the literature, and from the comparison with NGC3379 ([2], blue line) we note clear similarities due to the presence of diffuse shells in both systems.

We have also studied the fainter ETGs in the one square degree of the OmegaCAM field: NGC 4434, NGC 4464, NGC 4467, and VCC 1199, including the dwarf irregular, UGC 7636 in the proximity of the giant NGC 4472, reaching an even larger depth for these systems.

The (g-i) color profiles show an indication that for $r>3 r_{e}$ a very negative colour gradient develops in some galaxies, which apparently vanishes at $r \simeq 8 r_{e}$ (see Fig 3).

\subsection{2-dimensional model}

In order to enlighten possible larger substructures, a 2-dimensional elliptical model of NGC4472 best fitting the azimuthally averaged isophotes has been produced using the IRAF task BMODEL. Fig. 4 plot the difference between the original $g$ band image and the model. This residual map shows a clear asymmetry in the nuclear region and some

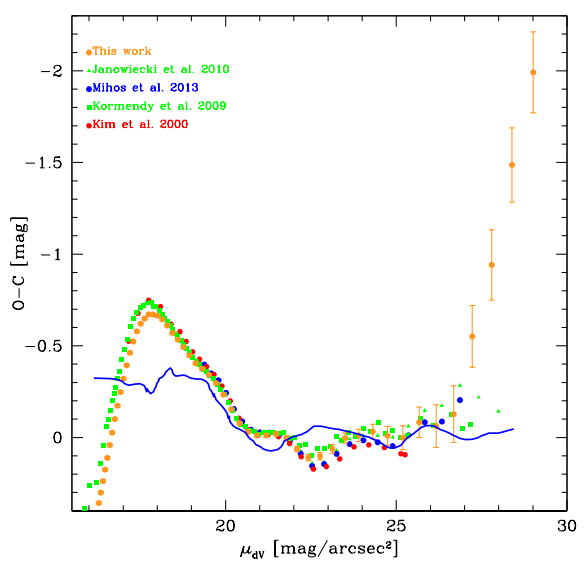

Fig. $2(\mathrm{O}-\mathrm{C})$ residuals of mean profiles from a best fitting $r^{1 / 4}$ model used only to remove the main gradient and make comparisons easier. The blue solid line plots the O-C residuals for the East-West photometric cross-section of the standard elliptical galaxy NGC3379 from [2]. diffuse features such as a tail associated with the dwarf irregular galaxy UGC 7636 interacting with NGC 4472, as well as the presence of concentric shells and fans of material (white contours), also identified by [1].

\section{Conclusions}

We have presented the VST Early-type Galaxy Survey (VEGAS), currently ongoing with VST/OmegaCAM (PI: M. Capaccioli). In particular, we present the deep observations in two bands ( $g$ and $i$ ), collected with the VST/OmegaCAM, for NGC4472. The surface brightness profiles of NGC 4472 reach a depth of $27.5 \mathrm{mag} / \mathrm{arcsec}^{2}$ in $g$ band and $26 \mathrm{mag} / \operatorname{arcsec}^{2}$ in $i$ band, comparably to previous deep studies (see Fig. 2). This depth allowed us to spot deviations from a simple de Vaucouleurs profile and in particular a change of slope at $a \sim 14^{\prime} .2$ (see Fig. (1) that we have associated to the presence of a decoupled ICL component which was not detected in previous analyses.

Here we stress that the simple inspection of the deep surface brightness profile of NGC 4472, clearly shows the presence of a diffuse component

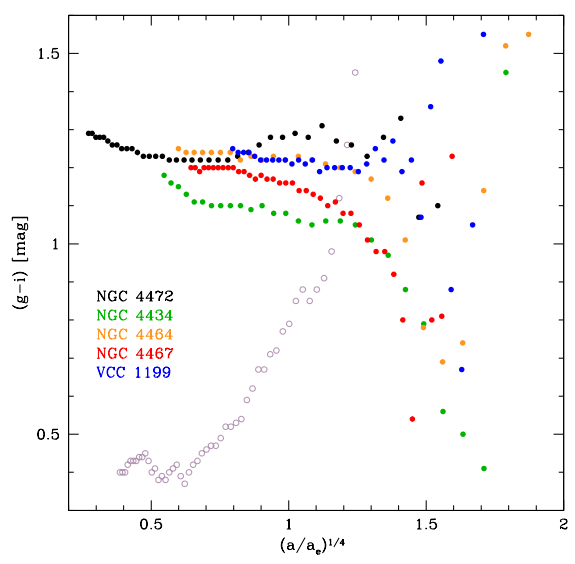

Fig. 3 Left-Azimuthally averaged light profiles in the $g$ band for the five ETGs of this paper scaled to their effective parameters. Right-Assembly of the $(g-i)$ color profiles for the five ETGs and for the interacting system UGC 7636. 
starting to dominate at $\mu_{g} \sim 26.5 \mathrm{mag} / \operatorname{arcsec}^{2}$ (see Fig. 1), which is compatible with the typical values at which [9] have observed change of slope induced by the ICL in a series of stacked galaxy clusters.

We notice that the trend of the residuals of the luminosity profiles of NGC 4472 with respect to an $r^{1 / 4}$ best fitting model has some striking analogies with the similar curve for NGC 3379 ([2]). Besides a bright extended core, we find evidence for a wavy pattern possibly associated with shells of diffuse material. The presence of such shells also stand out very clear from the 2-D residual map.

We have also studied the fainter ETGs in the one square degree of the OmegaCAM field: NGC 4434, NGC 4464, NGC 4467, and VCC 1199, including the dwarf irregular, UGC 7636 in the proximity of the giant galaxy NGC 4472. For all these systems we have highlighted the presence of some substructures defined as deviations from a simple de Vaucouleurs (1948) best fit profile. These deviations are associated to strong varying values of the ellipticity and P.A. as well as $a_{4}$ and $b_{4}$ parameters, suggesting the presence of some substructures.

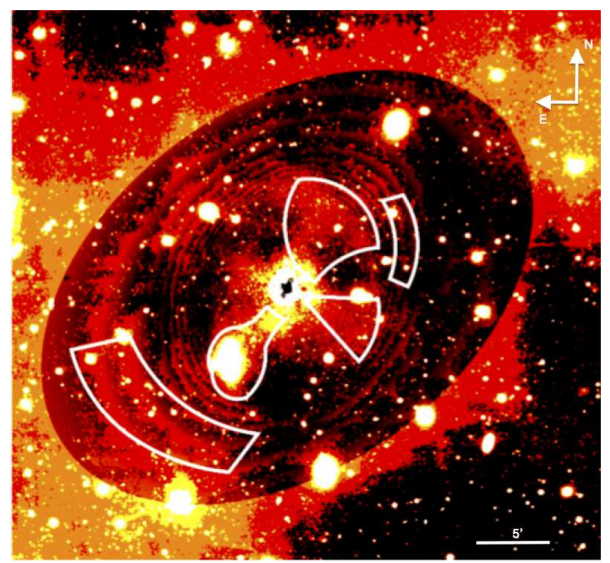

Fig. 4 NGC 4472 . Zoom $(37 \times 35$ arcmin $)$ of the mediansmoothed residual image. Superimposed white contours are 1) the tail connecting UGC 7636 to the giant ETG, and 2) shells and fan of material identified by [1] and visible in our residual image.
The color profiles show an indication that for $r>3 r_{e}$ a very negative colour gradient develops in some galaxies, which apparently vanishes at $r \simeq 8 r_{e}$. In a forthcoming paper (Capaccioli et al., in preparation) we will show that our results are basically unaffected in their qualitatively conclusions by the extended wings of the PSF (scattered light).

To conclude, this paper illustrates the performance and the accuracy achieved with the VST/OmegaCAM to produce surface photometry of early-type galaxies, also in very extreme conditions. For the case of NGC 4472 the presence of an extended halo around the giant galaxy, reaching the edge of the one square degree field of view, has allowed us to fully test the procedure for data reduction and background subtraction. The results obtained with our observations are comparable for accuracy to the collection of observations gathered from different telescopes (see [7]).

In the future we expect to implement a more variegate surface analysis including a wider set of photometric law in order to characterize the SB measurements in a larger sample of galaxies and thus discuss results in the context of galaxy formation theories.

\section{References}

1. Arrigoni Battaia, F., Gavazzi, G., Fumagalli, M., Boselli, A., Boissier, S., Cortese, L., et al. 2012, A\&A, 543, AA112

2. de Vaucouleurs, G., \& Capaccioli, M. 1979, ApJS, 40, 699

3. Ferrarese, L., Côté, P., Jordán, A., Peng, E. W., Blakeslee, J. P., Piatek, S., et al. 2006, ApJS, 164, 334

4. Gonzalez, A. H., Zabludoff, A. I., \& Zaritsky, D. 2005, ApJ, 618, 195

5. Janowiecki, S., Mihos, J. C., Harding, P., Feldmeier, J. J., Rudick, C., Morrison, H. 2010, ApJ, 715, 972

6. Kim, E., Lee, M. G., \& Geisler, D. 2000, MNRAS, 314,307

7. Kormendy, J., Fisher, D. B., Cornell, M. E., \& Bender, R. 2009, ApJS, 182, 216

8. Mihos, J. C., Harding, P., Rudick, C. S., \& Feldmeier, J. J. 2013, ApJL, 764, L20

9. Zibetti, S., White, S. D. M., Schneider, D. P., \& Brinkmann, J. 2005, MNRAS, 358, 949 